\title{
Mid-Term Load Forecasting Based on Grey Neural Network Corrector Model
}

\author{
Chao Yang ${ }^{1}$, Yunliang Wang ${ }^{2}$ \\ ${ }^{1}$ School of Electrical Engineering of TIANJIN UNIVERSITY OF TECHNOLOGY, 300384, China \\ ${ }^{2}$ Tianjin Key Laboratory for Control Theory \& Application in Complicated System, Tianjin University \\ of Technology, 300384, China
}

Keywords: load forecasting; related factors; grey model; BP neural network

\begin{abstract}
A novel grey neural network corrector model is presented in this paper, because mid-term load forecasting is affected by many factors and has large research space. This method combines BP neural network with 3 kinds of grey models, and selects influential factors by grey related analysis method, and then adds the equal dimension and new information technology. The validity of the novel model can be tested by utilizing actual data of a certain area. Comparing with 3 kinds of grey models, the novel model can improve the accuracy of load forecasting results. Experimental results prove that this method is feasible and effective to mid-term load forecasting.
\end{abstract}

\section{Introduction}

Load forecasting is an important task of power system's planning department. Accurate load forecasting can make power grid work in an economic and reasonable condition, and it can also enhance the economic and social benefit. Mid-term load forecasting is aimed at annual load value, and results have great value to power grid plan [1].

Currently, mid-term load forecasting methods are mainly divided into two stages. The first stage is traditional prediction technology which is represented by regression analysis method, trend extrapolation method and time series method [2]. The principle and structure of this kind of method is simple, and the forecasting speed is fast. But it cannot fit power load which has nonlinear, time-variable and uncertain characteristics. So the forecasting result is not ideal. The second stage is intelligent load forecasting technology. In this stage, a variety of methods emerge, such as expert system method, artificial neural network method, grey prediction method and so on [3]. These methods can fit nonlinear power load better than the first stage methods, and the forecasting result is more ideal. In these methods, artificial neural network method and grey prediction method are the most widely applied. In addition, a variety of new methods are also formed based on these two methods [4-6].

However, the load forecasting is a complicated nonlinear system which is affected by many factors [7-8]. Most of current methods just study on the power load data, but lack the consideration of the influential factors. These single fixed models will produce large error if regular pattern of power load changes. Power system is a highly complicated nonlinear system. In other words power system itself is a grey system, and then load forecasting can be regarded as grey prediction in essence. In this paper, a grey neural network corrector model has been established, which considers of the influential factors. It combines neural network with $\operatorname{GM}(1,1)$ model, $\mathrm{GM}(1, \mathrm{~N})$ model and $\mathrm{GM}(0, \mathrm{~N})$ model. This method not only considers the influential factors, but also has the advantage of grey prediction method that requires less data to model and has higher accuracy. Moreover, the new model also has the advantage of artificial neural network method which can do with the nonlinear problem well, so the accuracy of forecasting result can be improved.

\section{Grey theory \& grey model}

Grey theory.The grey system theory considers that any random process can be regarded as grey process. The grey process finds data's regular pattern by dealing with original data. This method reduces the randomness of the original data and obtains a new sequence. Regular pattern of the new 
sequence is strengthened. Grey theory has three kind of important models, which are $\operatorname{GM}(1,1)$ model, $\mathrm{GM}(1, \mathrm{~N})$ model and $\mathrm{GM}(0, \mathrm{~N})$ model.

GM(1,1) model. GM(1,1) model uses 1-AGO to do with the original data sequence. It is composed of first-order differential equation and has only one variable. GM(1,1) model is given as follow:

$$
\hat{x}^{(1)}(k+1)=\left[x^{(0)}(1)-\frac{u}{a}\right] e^{-a k}+\frac{u}{a} \quad(k=1,2, \cdots)
$$

$\mathbf{G M}(\mathbf{1 , N})$ model.Assuming there are several variables named $x_{1}, x_{2}, \ldots, x_{n}$. Where, $x_{1}$ is main factor, the others are influential factors. These variables construct several sequences. Using 1-AGO to do with these sequences, new sequences are generated. Then these new sequences construct first-order grey differential equation. $\mathrm{GM}(1, \mathrm{~N})$ model is given as follow:

$$
\hat{x}_{1}^{(1)}(k+1)=\left[x_{1}^{(0)}(1)-\frac{1}{a} \sum_{i=2}^{n} b_{i} x_{i}^{(1)}(k+1)\right] e^{-a k}+\frac{1}{a} \sum_{i=2}^{n} b_{i} x_{i}^{(1)}(k+1)
$$

$\operatorname{GM}(\mathbf{0 , N})$ model.GM(0,N) model is based on new sequences which are generated from original data sequences by 1 -AGO. $\mathrm{GM}(0, \mathrm{~N})$ model is given as follow:

$$
x_{1}^{(1)}(k)=a+b_{2} x_{2}^{(1)}(k)+b_{3} x_{3}^{(1)}(k)+\cdots+b_{n} x_{n}^{(1)}(k)
$$

Grey related analysis method.Grey related analysis method is used to analyze related degree of all factors in the system. Assuming there are several factors named $x_{1}, x_{2}, \ldots, x_{n}$. Where, $x_{1}$ is main factor. Computing related degree between $x_{i}$ and $x_{1}$ respectively, this paper names it $r_{i}$. The larger value of $r_{i}$ is, the greater factor $x_{i}$ affects the main factor $x_{1}$.

Equal dimension and new information technology.Grey forecasting method can only get high precision of few coming years' forecasting data. Equal dimension and new information technology updates historical data of model continuously and makes the dimension of model's historical data be equal. And this method does improve the precision of forecasting results.

\section{BP Neural Network}

BP neural network usually takes three layer network structures. By fitting the simple nonlinear function several times, BP neural network can approximate to any complex function. Usually Sigmoid function is selected as the transfer function:

$$
f(x)=\frac{1}{1+e^{-x}}
$$

\section{Grey neural network corrector model steps}

This paper establishes Grey neural network corrector model which considers related factors. It uses $\mathrm{GM}(1,1)$ model, $\mathrm{GM}(1, \mathrm{~N})$ model and $\mathrm{GM}(0, \mathrm{~N})$ model to forecast data respectively. It also adds the equal dimension and new information technology to refresh historical data, and corrects the forecasting data by neural network at last. The specific steps of the Grey neural network corrector model (RGM-NNC model) are as follows:

Step1: Firstly, the dimension of historical data should be determined. According to a large number of experiments, the forecasting result is the best when dimension of historical data is 7 . It is assumed the initial year value is $y$, and the data of power load and related factors that from $y$ to $y+6$ will be used as historical input data.

Step2: Analysis the grey related degree between load and each related factor. The related factor which owns the largest grey related degree is retained in this step.

Step3: Using GM(1,1) model which does not consider related factors to forecasting power load. And then using $\operatorname{GM}(1, N)$ model and $\operatorname{GM}(0, N)$ model which are based on related factors to forecasting power load respectively. Here these three models' forecasting years are $y, y+1, \ldots, y+7$.

Step4: Selecting three models' forecasting results of years from $y$ to $y+6$ as neurons of neural 
network's input layer. The actual historical load data are used as expected value to adjust neural network's weight. In order to convert data to $[0,1]$, the data of input layer should be normalized:

$$
\bar{x}_{i}=\frac{x_{i}-x_{\min }}{x_{\max }-x_{\min }}
$$

Where, $x_{i}$ represents input or output data, $x_{\min }$ represents the minimum value of data, $x_{\max }$ represents the maximum value of data.

Step5: This paper uses three-layer neural network structure and selects the Sigmoid function as transfer function. Calculating the number of neurons in the hidden layer by empirical formula:

$$
t=\sqrt{p+q}+c
$$

Where, $t$ is the number of nodes in hidden layer, $p$ is the number of nodes in the input layer, $q$ is the number of nodes in the output layer, $c$ is a constant integer among [1,10]. According to a large number of experiments when $c$ is 10 , the results are the best.

Step6: After the neural network has been trained, using the forecasting results of three kinds of models in the $y+7$ year as the input layer's neurons of the neural network. After the neural network finishes its calculation, the corrected forecasting data can be obtained. Dealing with the corrected forecasting data by reduction treatment, and then load forecasting value in the year of $y+7$ is obtained.

Step7: If the forecasting process has reached the target year, it will go to step 8. Otherwise, the actual load data and related factors of the year $y+7$ will be imported into the historical input data, and the historical input data of the year $y$ will be removed at the same time. In this way, the dimension of the input data can be fixed at 7 . Then assume the value of $y$

$$
y=y+1
$$

And then the process will go to step 2 .

Step8: The process has reached the target year, and it is ended.

\section{Experimental results analysis}

In this paper, the experiment is set up by software that Microsoft Visual $\mathrm{C}++6.0$ and MATLAB2010. In order to demonstrate the effectiveness of the RGM-NNC model, the sample which is a certain area's power load data and related factors from 2000 to 2012 year is selected. This paper adopts the regional GDP, the first industry additional value, the second industrial additional value and the regional GDP per capita as related factors. Data are shown in Table 1.

Table 1: The actual power load value and related factors value of a certain area

\begin{tabular}{cccccc}
\hline Year & $\begin{array}{c}\text { Actual } \\
\text { power load } \\
\text { value }\left(10^{8}\right. \\
\text { KWh })\end{array}$ & $\begin{array}{c}\text { Regional GDP } \\
\left(10^{8} \text { Yuan }\right)\end{array}$ & $\begin{array}{c}\text { The first } \\
\text { industry } \\
\text { additional } \\
\text { value } \\
\left(10^{8} \text { Yuan }\right)\end{array}$ & $\begin{array}{c}\text { The second } \\
\text { industrial } \\
\text { additional } \\
\text { value } \\
\left(10^{8} \text { Yuan }\right)\end{array}$ & $\begin{array}{c}\text { Regional } \\
\text { GDP per } \\
\text { capita } \\
\text { (Yuan per } \\
\text { capita })\end{array}$ \\
\hline 2000 & 559.45 & 4771.17 & 76.68 & 2207.63 & 30047 \\
2001 & 592.98 & 5210.12 & 78 & 2403.18 & 31799 \\
2002 & 645.71 & 5741.03 & 79.68 & 2622.45 & 33958 \\
2003 & 745.97 & 6694.23 & 81.02 & 3209.02 & 38486 \\
2004 & 821.44 & 8072.83 & 83.45 & 3892.12 & 44839 \\
2005 & 921.97 & 9247.66 & 90.26 & 4381.2 & 49649 \\
2006 & 990.15 & 10572.24 & 93.81 & 4969.95 & 54858 \\
2007 & 1072.38 & 12494.01 & 101.84 & 5571.06 & 62041 \\
2008 & 1138.22 & 14069.87 & 111.8 & 6085.84 & 66932 \\
2009 & 1153.38 & 15046.45 & 113.82 & 6001.78 & 69164 \\
2010 & 1295.87 & 17165.98 & 114.15 & 7218.32 & 76074 \\
2011 & 1339.62 & 19195.69 & 124.94 & 7927.89 & 82560 \\
2012 & 1353.45 & 20181.72 & 127.8 & 7854.77 & 85373 \\
\hline
\end{tabular}


The data from 2000 to 2006 year in Table 1 is used as the original data. After forecasting the power load in the next year of the original data, this paper uses equal dimension and new information technology to renew the original data. The forecasting results of grey models and RGM-NNC model are shown in Table 2.

Table 2: Forecasting results of models

\begin{tabular}{|c|c|c|c|c|c|}
\hline Year & $\begin{array}{l}\text { Actual power load } \\
\text { value }\end{array}$ & $\mathrm{GM}(1,1)$ & $\mathrm{GM}(1, \mathrm{~N})$ & $\mathrm{GM}(0, N)$ & RGM-NNC \\
\hline 2007 & 1072.38 & 1115.69 & 1148.87 & 1151.39 & 1038.73 \\
\hline 2008 & 1138.22 & 1198.74 & 1263.26 & 1272.78 & 1133.78 \\
\hline 2009 & 1153.38 & 1255.28 & 1329.71 & 1343.24 & 1185.12 \\
\hline 2010 & 1295.87 & 1271.94 & 1343.34 & 1355.08 & 1213.27 \\
\hline 2011 & 1339.62 & 1358.79 & 1404.21 & 1417.76 & 1364.21 \\
\hline 2012 & 1353.45 & 1428.11 & 1391.51 & 1380.99 & 1399.84 \\
\hline
\end{tabular}

In order to make the forecasting results of four models more intuitive, this paper uses MATLAB2010 to simulate. The curves of the actual power load value and models' forecasting results are shown in Figure 1.
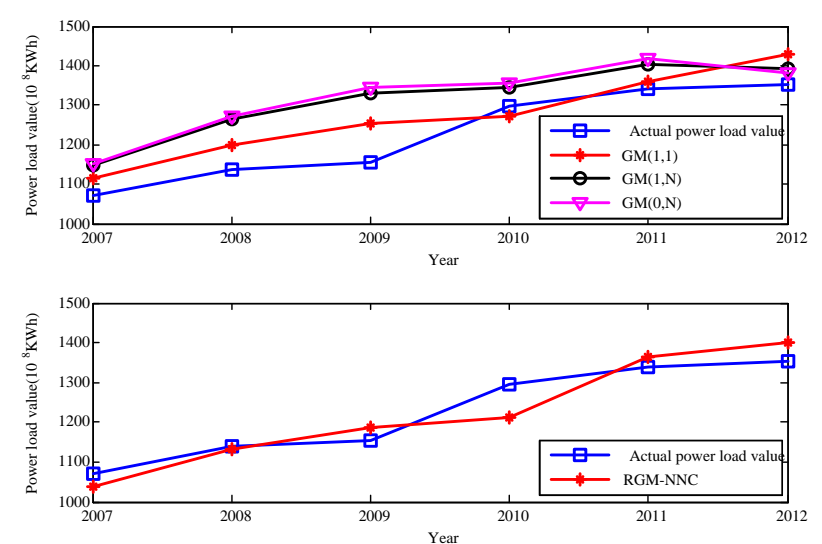

Fig.1: The curves of the actual power consumption and forecasting results of models

From Table 2 and Fig. 1 it can be seen that comparing with the forecasting curves of $\operatorname{GM}(1,1)$, $\operatorname{GM}(1, N)$ and $\operatorname{GM}(0, N)$, the forecasting curve of RGM-NNC is much closer to the curve of actual power consumption. Because the equal dimension and new information technology is added into the RGM-NNC, the RGM-NNC can capture the changing trend of the actual power consumption better. For example, the forecasting result of RGM-NNC has obvious difference with the actual power consumption in the year of 2010, and the difference between the forecasting result of RGM-NNC and the actual power consumption decreases obviously in the next year.

The relative error of models' forecasting results is shown in Table 3. The average absolute error of models' forecasting results is shown in Table 4.

Table3: The relative error of models' forecasting results

\begin{tabular}{lllll}
\hline Year & GM(1,1) & GM(1,N) & GM $(0, N)$ & RGM-NNC \\
\hline 2007 & -0.0403907 & -0.0713304 & -0.0736817 & 0.0313784 \\
2008 & -0.053175 & -0.109852 & -0.118219 & 0.0038979 \\
2009 & -0.0883529 & -0.152883 & -0.16461 & -0.0275222 \\
2010 & 0.0184668 & -0.0366348 & -0.0456919 & 0.063739 \\
2011 & -0.0143083 & -0.0482171 & -0.0583308 & -0.0183585 \\
2012 & -0.0551623 & -0.028123 & -0.020345 & -0.0342752 \\
\hline
\end{tabular}

Table4: The average absolute error of models' forecasting results

\begin{tabular}{lllll}
\multicolumn{5}{c}{$10^{8} \mathrm{KWh}$} \\
\hline & $\mathrm{GM}(1,1)$ & $\mathrm{GM}(1, \mathrm{~N})$ & $\mathrm{GM}(0, \mathrm{~N})$ & RGM-NNC \\
\hline MAE & 53.9169 & 87.9984 & 94.7201 & 37.2351 \\
\hline
\end{tabular}


From Table 3 it can been found that the absolute value of the relative error of GM(1,1)'s forecasting results is between $1.43083 \%$ and $8.83529 \%$, and the absolute value of the relative error of $\mathrm{GM}(1, \mathrm{~N})$ 's forecasting results is between $2.8123 \%$ and $15.2883 \%$, and the absolute value of the relative error of $\mathrm{GM}(0, \mathrm{~N})$ 's forecasting results is between $2.0345 \%$ and $16.461 \%$, but the absolute value of the relative error of RGM-NNC's forecasting results is between $0.38979 \%$ and $6.3739 \%$. Thus the experimental results indicate that the error of RGM-NNC is much smaller than GM(1,1), $\mathrm{GM}(1, \mathrm{~N})$ and $\mathrm{GM}(0, \mathrm{~N})$. In addition, from Table 4 it can be concluded that comparing with $\mathrm{GM}(1,1)$, $\mathrm{GM}(1, \mathrm{~N})$ and $\mathrm{GM}(0, \mathrm{~N})$, the MAE of RGM-NNC's forecasting results reduces $1668180000 \mathrm{KWh}$, $5076330000 \mathrm{KWh}$ and $5748500000 \mathrm{KWh}$ respectively. So the accuracy of forecasting results can be improved by the RGM-NNC.

\section{Conclusions}

Considering the actual situation, power load is influenced by many factors. This paper uses $\operatorname{GM}(1, N), \operatorname{GM}(0, N)$ and the classical $\mathrm{GM}(1,1)$ to forecast power load respectively , and then utilizes BP neural network to correct forecasting results. From the forecasting results, it can be concluded that this novel composite model can capture the trend of power load value in the future better, and the accuracy of forecasting results can be improved obviously, so the method is feasible and effective.

\section{References}

[1] Niu Dongxiao, Cao Shuhua, Lu Jianchang, Zhao Lei. Electric power load forecasting technology and its application. Beijing: China Electric Power Press, 2009.

[2] ZHOU Chao, XING Wei-yang, LI Yu-long. Summarization on Load Forecasting Method of Electrical Power System. Journal of Power Supply, 2012, (6): 32-39.

[3] ZHANG Jian-mei, ZHOU Bu-xiang, LIN Nan, ZHANG Qin, CHEN Jie. Prediction of Mid-long Term Load Based on Gray Elman Neural Networks. Proceedings of the CSU-EPSA, 2013, 25(4): 145-149.

[4] ZHOU De-qiang, WU Ben-ling. Optimization and Power Load Forecasting of Gray BP Neural Network Model. Power System Protection and Control, 2011, 39(21): 65-69.

[5] QIN Yu-yi, LING Liang, SONG Yong-hua, WEI Shao-yan. Short-term Load Forecasting Based on Grey Model and ANN. Modern Electric Power, 2009, 26(2): 1-4.

[6] LI Xiao-xia, ZHANG Pei-jun. Medium-long Power Load Forecasting Based on Improved Grey BP Model. Proceedings of the 1st International Workshop on Education Technology and Computer Science, ETCS 2009, 2009, 2: 366-368.

[7] NIU Dong-xiao, ZHANG Tong-tong, CHEN Li-rong, ZHANG Bo. Grey Load Forecasting Models Based on Relational Analysis of Multi-factor. Journal of North China Electric Power University, 2006, 33(3): 90-92.

[8] GU Xi-hua, XING Mian, NIU Dong-xiao. Multifactor-influenced Combined Gray Neural Network Models for Power Load Forecasting. East China Electric Power, 2006, 34(7): 6-9. 